\title{
Correspondence
}

\section{Spectral Arrangement and other Topics in First-Order Bandpass Sampling Theory}

Jianhua Liu, Xiyuan Zhou, and Yingning Peng

\begin{abstract}
A problem of first-order bandpass sampling is that the indifferent disposition of the baseband spectrum may cause inconvenience in practical applications. To overcome it, we modify the first-order bandpass sampling theory according to the spectral arrangement of the sampled signal. We consider two situations on the arrangement of the shifted replicas of the bandpass signal spectrum: the normal and the inverse placement of the spectrum. The positive spectrum of the bandpass signal is placed in the lowest positive part of the sampled signal in the normal placement situation and in the lowest negative part in the inverse situation. The sampling frequencies are formulated in a cluster of lower and upper bounds in both situations, and robust sampling frequencies are given. Topics of the modified theory related to applications are addressed.
\end{abstract}

Index Terms-Bandpass signals, first-order sampling, sampling theory.

\section{INTRODUCTION}

Bandpass signals are frequently encountered in theoretical research and engineering, and the first-order sampling theory of bandpass signals has drawn considerable interest in a variety of areas such as communications, radar, sonar, measurement, optics, etc.

A real bandpass signal $f(t)$ can be defined as follows. Let the real positive numbers $\underline{\omega}_{C}$ and $2 \omega_{B}$ satisfying $\omega_{C} \geq \omega_{B}$ represent the central frequency and bandwidth of the bandpass signal $f(t)$, respectively, and let $\omega_{L}=\omega_{C}-\omega_{B}$ and $\omega_{U}=\omega_{C}+\omega_{B}$ denote the lower and upper cutoff frequencies, respectively. Assume that $S(\omega)$ is the spectrum of a baseband complex signal $s(t)$ that satisfies

$$
|S(\omega)|=0, \quad \forall|\omega| \geq \omega_{B} .
$$

The spectrum of $f(t)$ is defined as

$$
F(\omega)=S\left(\omega-\omega_{C}\right)+S^{*}\left(-\omega-\omega_{C}\right)
$$

as shown in Fig. 1(a), where $(\cdot)^{*}$ stands for complex conjugate.

The band position is defined as $\omega_{L}$ normalized by $2 \omega_{B}$. A special case is integer band positioning that occurs when $\omega_{L}$ and $2 \omega_{B}$ satisfies $\omega_{L}=2 c \omega_{B}$ for $c=0,1,2, \cdots$. It is obvious that $c=0$ is the lowpass case, and hence, the lowpass signal is just a special case of a bandpass signal.

First-order sampling is characterized by a uniform sampling interval; it is different from the second-order sampling [1] and other nonuniform sampling [2]. Kohlenberg [1] showed that for first-order sampling, the

Manuscript received September 7, 1997; revised February 23, 2001. The associate editor coordinating the review of this paper and approving it for publication was Dr. Ali H. Sayed.

J. Liu was with Communications, Telemetry, and Telecontrol Research Institute, Shijiazhuang, China, and the Department of Electronic Engineering, Tsinghua University, Beijing, China. He is now with the Department of Electrical and Computer Engineering, University of Florida, Gainsville, FL 32611 USA, on leave from the School of Electrical and Electronic Engineering, Nanyang Technological University, Singapore.

$\mathrm{X}$. Zhou is with the Communications, Telemetry, and Telecontrol Research Institute, Shijiazhuang, China.

Y. Peng is with the Department of Electronic Engineering, Tsinghua University, Beijing, China.

Publisher Item Identifier S 1053-587X(01)03881-8.
Nyquist criterion $\omega_{S} \geq 2 \omega_{U}$ (where $\omega_{S}$ is the sampling frequency) [3] only holds for the equality in the case of integer band positioning, and for other cases, the criterion can be attained only by nonuniform sampling.

Several text books, such as [4], state that for general cases, the minimum sampling frequency is

$$
\omega_{S}=\frac{2 \omega_{U}}{\left\lfloor\frac{\omega_{U}}{2 \omega_{B}}\right\rfloor}
$$

where $\lfloor x\rfloor$ stands for the largest integer not bigger than $x$.

Actually, (3) only gives the lowest sampling frequency, and aliasing may occur for higher sampling frequencies. Gaskell [5] modified (3) to yield the range of sampling frequencies for which no aliasing occurs

$$
\frac{2 \omega_{U}}{n} \leq \omega_{S} \leq \frac{2 \omega_{L}}{n-1}
$$

where $\underline{n}$ is an integer given by $1 \leq n \leq \omega_{U} /\left(2 \omega_{B}\right)$.

Although the aliasing problem is solved perfectly in (4), the indifferent disposition of the baseband spectrum may cause inconvenience in practical applications such as digital demodulation of single sideband (SSB) signal because the placement of the baseband spectrum may be inverted. We have therefore modified the sampling conditions for obtaining the normal and inverse spectral placement situation in [6] The essence of our former results are presented here.

Vaughan et al. [7] noted that frequencies satisfying the equality in (4) exhibit high sensitivity to change and improved the theorem of Gaskell by adding a guardband to make the sampling more robust. They also suggested obtaining the sampling frequency as

$$
\omega_{S}=\frac{\omega_{U}}{n}+\frac{\omega_{L}}{n-1}
$$

to increase the robustness

Equation (5) implies asymmetric sampling frequency tolerances provided that the guardbands are symmetric. We propose robust sampling frequency formulas in the above mentioned two cases to guarantee symmetric sampling frequency tolerances in the case of symmetric filter stopband attenuation.

In addition, several important topics of the modified first-order bandpass sampling theory related to applications such as phase-difference extraction, effect of time delay, and effect of the sampling stability are addressed.

\section{THEORY OF FIRST-ORDER BANDPASS SAMPLING}

Demodulating theory of first-order bandpass sampling is modified in this section based on the observation that the sampled baseband signal may have two kinds of spectral structure according to the sampling frequency chosen.

\section{A. First-Order Sampling}

First-order sampling of a bandpass signal $f(t)$ is implemented by multiplying $f(t)$ by a periodic train of uniform impulses $\delta_{T}(t)$ with interval $T$ (the sampling interval). Letting $f_{S}(t)$ and $F_{S}(\omega)$ stand for the sampled (discrete) signal and its spectrum, respectively, we have

$$
f_{S}(t)=\sum_{k=-\infty}^{\infty} f(t) \cdot \delta(t-k T)
$$




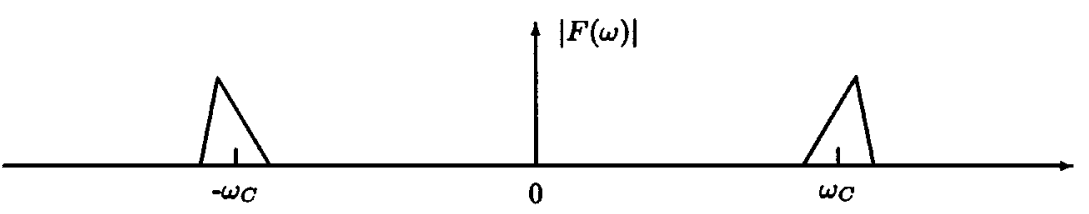

(a)

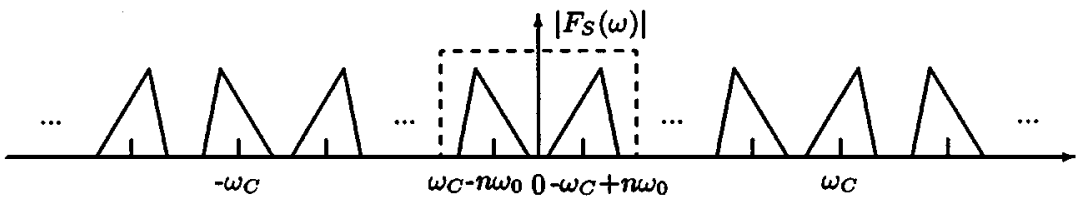

(b)

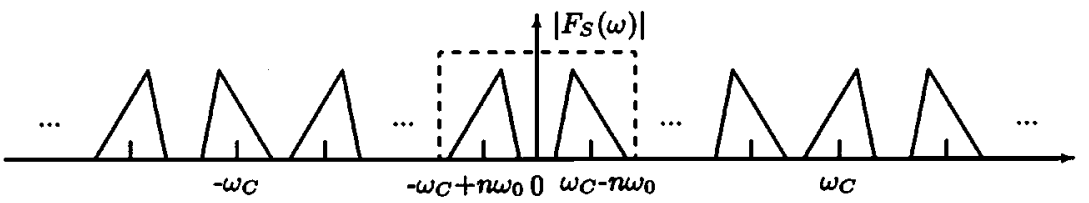

(c)

Fig. 1. Spectrum of signals. (a) Bandpass signal. (b) Sampled signal of normal spectral placement. (c) Sampled signal of inverse spectral placement.

and

$$
F_{S}(\omega)=\frac{1}{T} \sum_{n=-\infty}^{\infty} F\left(\omega-n \omega_{S}\right)
$$

We can see from (7) that $F_{S}(\omega)$ consists of replicas of $F(\omega)$ shifted by $n \omega_{S}$, where $n=0, \pm 1, \pm 2, \cdots 1$ To demodulate correctly from $F_{S}(\omega)$, it is essential that there is no aliasing in $F_{S}(\omega)$. As $F(\omega)$ has two separated parts of spectrum, $F_{S}(\omega)$ must be an ordered placement of $F(\omega)$, i.e., the positive and negative parts of $F\left(\omega-n \omega_{S}\right), n=0$, $\pm 1, \pm 2, \cdots$ should be placed on the $\omega$ axis alternatively.

\section{B. Spectral Placement}

In many cases, the signal of interest obtained by first-order sampling of a bandpass signal is the signal associated with the pair of the lowest spectral bands as shown in the dashed-lined box in Fig. 1(b) and (c).

If the sampled signal is required to possess the same spectral structure as the bandpass signal, the positive spectrum of the bandpass signal should be placed in the lowest positive part, as shown in Fig. 1(b). We call this situation the normal spectral placement.

In some circumstances, it is not necessary for the sampled signal to possess the same spectral structure as the bandpass signal; in this case, the positive spectrum of the bandpass signal can be placed in the lowest negative part, as shown in Fig. 1(c). We call it the inverse spectral placement.

The inverse spectral placement is necessary in some practical situation, for example, when the bandpass signal possesses the inverse spectral structure after processing in an intermediate frequency (IF) stage.

The ranges of sampling frequencies that guarantee that no aliasing occurs can be obtained from (4) by requiring $n$ to be odd for normal spectral placement and even for inverse spectral placement. To highlight the fact that we have normal and inverse placement of the baseband spectrum, we give a complete derivation of sampling frequencies below.

${ }^{1}$ A positive $n$ means right shift, and a negative one means left.

\section{Sampling Frequencies for Normal Placement}

As the positive and negative parts of the bandpass signal spectrum should be placed alternatively when sampled, the necessary condition

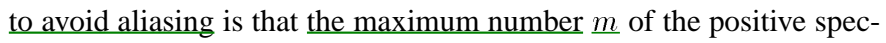
trum of the bandpass signal placed in the interval $\left[0, \omega_{C}-\omega_{B}\right]$ should be limited as

$$
m=\left\lfloor\frac{\omega_{C}-\omega_{B}}{4 \omega_{B}}\right\rfloor .
$$

When the integer $\underline{n}$ (we call it frequency shift coefficient) $\underline{\text { satisfies }}$ $0 \leq n \leq m$, the $n$th left shift of the positive spectrum of $F(\omega)$ can be placed in the lowest positive part of the sampled signal. Because of the periodicity of $F_{S}(\omega)$, the aliasing will occur periodically if the sampling frequency is not carefully selected. To avoid the aliasing in the case of normal spectral placement, the upper limit of the sampling frequency is determined by locating the $2 n$th left shift of the positive spectrum of $F(\omega)$ to the right side of the negative part of $F(\omega)$ without aliasing

$$
\left(\omega_{C}-2 n \omega_{S}\right)-\left(-\omega_{C}\right) \geq 2 \omega_{B} .
$$

Thus, we have

$$
\omega_{S} \leq \frac{\omega_{C}-\omega_{B}}{n}
$$

and the lower limit of the sampling frequency is determined by locating the $2 n+1$ th left shift of the positive spectrum of $F(\omega)$ to the left side of the negative part of $F(\omega)$ without aliasing

$$
\left(-\omega_{C}\right)-\left(\omega_{C}-(2 n+1) \omega_{S}\right) \geq 2 \omega_{B}
$$

and we have

$$
\omega_{S} \geq \frac{2\left(\omega_{C}+\omega_{B}\right)}{2 n+1}
$$

Therefore, the available range of the sampling frequencies is

$$
\frac{2\left(\omega_{C}+\omega_{B}\right)}{2 n+1} \leq \omega_{S} \leq \frac{\omega_{C}-\omega_{B}}{n} .
$$


As we know, the practical bandpass filters do not have infinite stopband attenuation; therefore, to reduce the aliasing to the minimum and to overcome the problem of asymmetric sampling frequency tolerances in the case of symmetric stopband attenuation on the two sides of the bandpass filter in (5), the robust sampling frequency should be chosen to put the negative spectrum of $F(\omega)$ in the center of the $2 n$th and $2 n+1$ th left shift of the positive one

$$
\left(\omega_{C}-2 n \omega_{S}\right)+\left(\omega_{C}-(2 n+1) \omega_{S}\right)=-2 \omega_{C}
$$

which means

$$
\omega_{S}=\frac{4 \omega_{C}}{4 n+1}
$$

The allowed areas of sampling frequencies determined by (11) and the robust sampling frequencies determined by (12) are shown in Fig. 2(a).

\section{Sampling Frequencies for Inverse Placement}

Similar to the situation of normal spectral placement, the necessary condition to avoid aliasing in the case of inverse spectral placement is that the maximum number $\underline{m}$ of the positive spectrum of the bandpass signal placed in the interval $\left[0, \omega_{C}+\omega_{B}\right]$ should be limited as

$$
m=\left\lfloor\frac{\omega_{C}+\omega_{B}}{4 \omega_{B}}\right\rfloor .
$$

When the frequency shift coefficient $\underline{n}$ satisfies $1 \leq n \leq m$, the $n$th left shift of the positive spectrum of $F(\omega)$ can be placed in the lowest negative part of the sampled signal. To avoid the aliasing in the case of the inverse spectral placement, the $2 n$th and $2 n-1$ th left shift of the positive spectrum of $F(\omega)$ should be located without any aliasing at the left and right side of the negative spectrum of $F(\omega)$, respectively, as

$$
\begin{aligned}
\left(-\omega_{C}\right)-\left(\omega_{C}-2 n \omega_{S}\right) & \geq 2 \omega_{B} \\
\left(\omega_{C}-(2 n-1) \omega_{S}\right)-\left(-\omega_{C}\right) & \geq 2 \omega_{B} .
\end{aligned}
$$

Therefore, the available range of the sampling frequency is

$$
\frac{\omega_{C}+\omega_{B}}{n} \leq \omega_{S} \leq \frac{2\left(\omega_{C}-\omega_{B}\right)}{2 n-1} .
$$

As above, the most robust sampling frequency can be obtained by putting the negative spectrum of $F(\omega)$ in the center of the $2 n-1$ th and $2 n$th left shift of the positive one

$$
\left(\omega_{C}-(2 n-1) \omega_{S}\right)+\left(\omega_{C}-2 n \omega_{S}\right)=-2 \omega_{C}
$$

that is

$$
\omega_{S}=\frac{4 \omega_{C}}{4 n-1}
$$

The allowed areas of sampling frequencies determined by (14) and the robust sampling frequencies determined by (15) are shown in Fig. 2(b).

\section{OTHER TOPICS OF THE SAMPLING THEORY}

We address several important topics of the modified first-order bandpass sampling theory related to applications such as phase-difference extraction, effect of time delay, and effect of the sampling stability in this section.

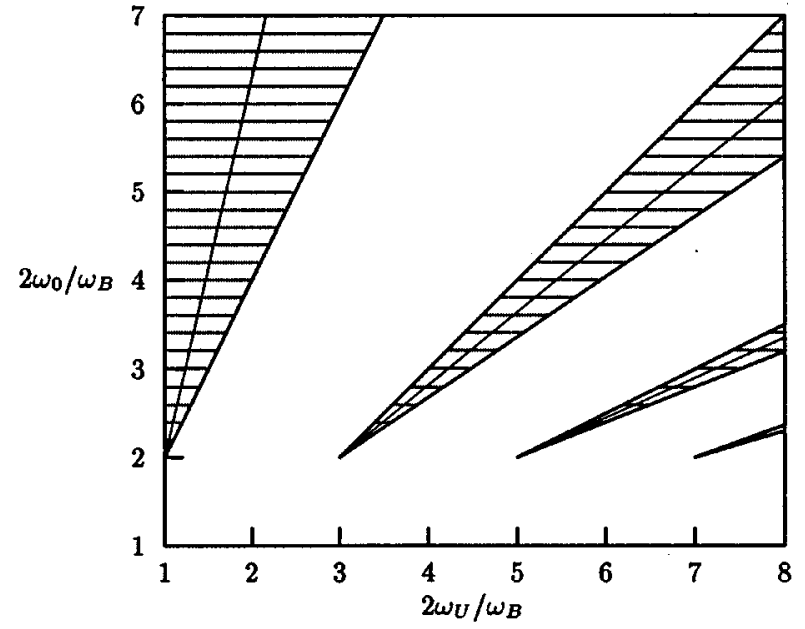

(a)

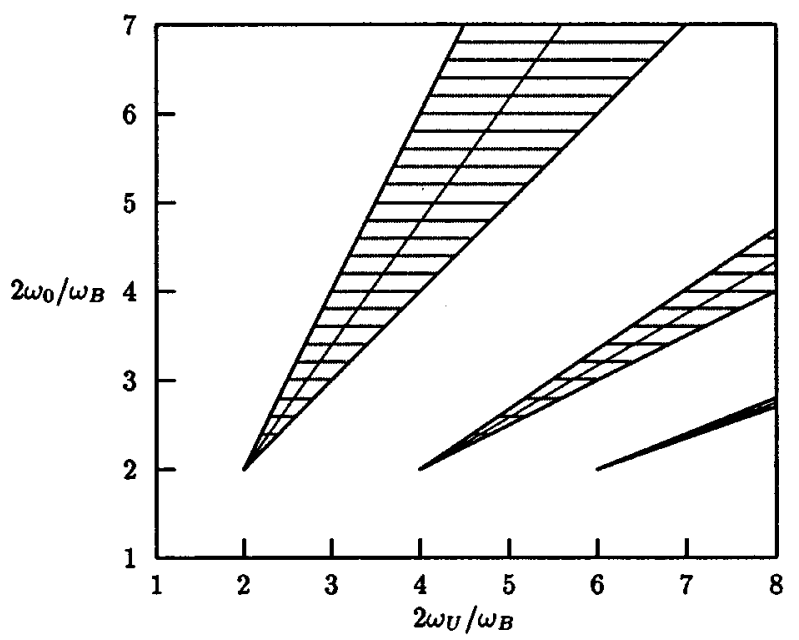

(b)

Fig. 2. Allowed areas of the sampling frequencies (dotted areas) and the robust sampling frequencies (thin lines). (a) Case of normal spectral placement. (b) Case of inverse spectral placement.

\section{A. Phase-Difference Extraction}

Consider two bandpass signals $f(t)$ and $g(t)$ with spectrum

$$
\begin{aligned}
& F(\omega)=S_{1}\left(\omega-\omega_{C}\right)+S_{1}^{*}\left(-\omega-\omega_{C}\right) \\
& G(\omega)=S_{2}\left(\omega-\omega_{C}\right)+S_{2}^{*}\left(-\omega-\omega_{C}\right)
\end{aligned}
$$

respectively, where $S_{1}(\omega)$ and $S_{2}(\omega)$ satisfy (1). Because of the conjugate symmetry, we may consider only the positive spectrum of the signal to calculate the phase difference. The phase difference between $f(t)$ and $g(t)$ is

$$
\theta(\omega)=\arctan F(\omega) G^{*}(\omega), \quad \omega \in\left[\omega_{L}, \omega_{U}\right]
$$

In the case of normal spectral placement, the phase difference between the sampled baseband signal is

$$
\begin{gathered}
\theta_{P}(\omega)=\arctan S_{1}\left(\omega-\omega_{C}-n \omega_{S}\right) S_{2}^{*}\left(\omega-\omega_{C}-n \omega_{S}\right), \\
\omega \in\left[\omega_{L}-n \omega_{S}, \omega_{U}-n \omega_{S}\right] .
\end{gathered}
$$


While in the case of inverse placement, the phase difference between the sampled baseband signal is

$$
\begin{gathered}
\theta_{I}(\omega)=-\arctan S_{1}\left(-\omega-\omega_{C}+n \omega_{S}\right) S_{2}^{*}\left(-\omega-\omega_{C}+n \omega_{S}\right) \\
\omega \in\left[n \omega_{S}-\omega_{U}, n \omega_{S}-\omega_{L}\right] .
\end{gathered}
$$

From the above equations, we know that $\theta_{P}$ is the same as $\theta$ and that $\theta_{I}$ is the inverse of $\theta$.

\section{B. Effect of Time Delay}

Obviously, the effect of time delay only exists in the phase. As above, we may consider only the positive spectrum of the bandpass signal to determine the effect. Suppose $f(t)$ is a bandpass signal with spectrum defined by (2) and $g(t)=f(t-\tau)$. The positive spectra of $f(t)$ and $g(t)$ are

$$
\begin{aligned}
& F(\omega)=S\left(\omega-\omega_{C}\right) \\
& G(\omega)=S\left(\omega-\omega_{C}\right) e^{-j \omega \tau} .
\end{aligned}
$$

In the case of normal spectral placement, the lowest positive spectra of the sampled signals are

$$
\begin{aligned}
& F_{0}(\omega)=S\left(\omega-\omega_{C}+n \omega_{S}\right) \\
& G_{0}(\omega)=S\left(\omega-\omega_{C}+n \omega_{S}\right) e^{-j\left(\omega+n \omega_{S}\right) \tau}
\end{aligned}
$$

where

$$
\omega \in\left[\omega_{C}-\omega_{B}-n \omega_{S}, \omega_{C}-\omega_{B}-n \omega_{S}\right] .
$$

Hence, the phase-difference between the sampled signals is

$$
\begin{array}{r}
\theta_{P}=\arctan F_{0}(\omega) G_{0}^{*}(\omega)=\left(\omega+n \omega_{S}\right) \tau \\
\omega \in\left[\omega_{L}-n \omega_{S}, \omega_{U}-n \omega_{S}\right] .
\end{array}
$$

In the case of inverse spectral placement, the lowest negative spectra of the sampled signals are the same as (21) and (22), and hence, the phase difference is

$$
\theta_{I}=-\left(\omega+n \omega_{S}\right) \tau, \quad \omega \in\left[\omega_{L}-n \omega_{S}, \omega_{U}-n \omega_{S}\right] .
$$

It should be noted that the ranges of $\omega$ in (23) and (24) are not the same because the sampling frequencies are different in the two cases.
Equations (23) and (24) are especially useful in the case of delayed sampling, which may occur in sampling several bandpass signals using one A/D or obtaining the desired phase shift for beamforming by controlling the sampling delay [8].

\section{Effect of Sampling Stability}

Vaughan $e t$ al. analyzed the effect of sampling stability thoroughly. We add a result of the effect of sampling stability on the precision of frequency analysis.

From the derivation of (11) and (14), we can see that an error $\Delta \omega_{S}$ on the sampling frequency will cause a total drift of

$$
\Delta \omega=n \Delta \omega_{S}
$$

in the lowest pair of spectrum of the sampled signal. This means that the analyzing error of the sampled baseband signal is $n$ times the sampling oscillator perturbance.

\section{CONCLUding REMARKS}

Demodulating theory of first-order bandpass sampling is modified in this correspondence based on the observation that the sampled baseband signal may have two kinds of spectral arrangement, i.e., the normal spectral placement and the inverse spectral placement, according to the sampling frequency chosen. The robust sampling is improved to have the same sampling frequency tolerances in two sides of the guardband.

In addition, we addressed several important topics of the modified first-order bandpass sampling theory related to applications such as phase-difference extraction, effect of time delay, and effect of the sampling stability in the context.

\section{REFERENCES}

[1] A. Kohlenberg, "Exact interpolation of band-limited functions," J. Appl. Phys., vol. 24, no. 12, pp. 1432-1436, Dec. 1953.

[2] A. J. Coulson, "A generalization of nonuniform bandpass sampling," IEEE Trans. Signal Process., vol. 43, pp. 694-704, Mar. 1995.

[3] H. Nyquist, "Certain topics in telegraph transmission theory," Trans. Amer. Inst. Elect. Eng., vol. 47, pp. 617-644, 1928.

[4] P. Z. Peebles, Jr., Communication System Principles. Reading, MA: Addison-Wesley, 1976.

[5] J. D. Gaskell, Linear Systems, Fourier Transforms, and Optics. New York: Wiley, 1978.

[6] J. H. Liu and X. Y. Zhou, "Spectrum arrangement and the generalized bandpass signal direct sampling theorem," in Proc. Int. Conf. Signal Process., vol. 1, Beijing, China, Oct. 1996, pp. 28-31.

[7] R. G. Vaughan, N. L. Scott, and D. R. White, "The theory of bandpass sampling," IEEE Trans. Signal Processing, vol. 39, pp. 1973-1984, Sept. 1991.

[8] J. H. Liu, Y. N. Peng, and L. S. Tian, "Digitized beamforming via delayed first-order bandpass sampling," in Proc. Int. Conf. Signal Process., vol. 1, Beijing, China, Oct. 1998, pp. 369-372. 